\title{
Les rapports mobilité/immobilité dans le cas de situations résidentielles spécifiques : retours et perspectives de recherche
}

Céline Bergeon

\section{CpenEdition}

Journals

Édition électronique

URL : https://journals.openedition.org/e-migrinter/249

DOI : 10.4000/e-migrinter.249

ISSN : 1961-9685

Éditeur

UMR 7301 - Migrinter

\section{Édition imprimée}

Date de publication : 1 septembre 2013

Pagination : 28-35

ISSN : 1961-9685

\section{Référence électronique}

Céline Bergeon, «Les rapports mobilité/immobilité dans le cas de situations résidentielles spécifiques : retours et perspectives de recherche », e-Migrinter [En ligne], 11 | 2013, mis en ligne le, consulté le 20 mai 2021. URL : http://journals.openedition.org/e-migrinter/249 ; DOI : https://doi.org/ 10.4000/e-migrinter.249 


\section{Les rapports mobilité/immobilité dans le cas de situations résidentielles spécifiques : retours et perspectives de recherche}

Céline Bergeon

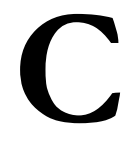

ette présentation s'articule autour

d'une discussion entre deux catégories de l'habitat - qui sont supposées introduire de 1' « instabilité résidentielle » - et la place de l'immobilité dans cette apparente et présupposée mobilité. Elle se nourrit d'une analyse faite dans le cadre de ma thèse portant sur la mobilité des Gens du voyage en France et en Belgique, ainsi que sur les questionnements élaborés dans le cadre de mes recherches post-doctorales. Enfin, la question des limites méthodologiques est traitée en dernière partie, à partir d'une réflexion sur les catégories (de personnes et de pratiques) préétablies qui peuvent orienter le recueil de données.
Dans un premier temps, j’aborderai la question des pratiques liées à l'habitat mobile afin de révéler les liens existant entre mobilité et immobilité et de déconstruire des catégories qui reposent essentiellement sur leur caractéristique de «mobile»: il y a ici une collusion entre type d'habitat et pratiques, et ces catégories se focalisent essentiellement sur la mobilité. Enfin dans un second temps, je me pencherai sur des pratiques liées à l'habitat précaire qui, de par son caractère supposé instable et éphémère, interroge cette notion d'immobilité. Enfin, l'aspect méthodologique est transversal à ces deux premiers points: il s'intéresse particulièrement à l'analyse des pratiques de l'immobilité quand les catégories sont conditionnées par la mobilité.

Partant d'une relecture de mes travaux et d'une discussion ébauchée dans mes perspectives de recherche, je souhaite voir la place de l'immobilité en contexte "d'instabilité résidentielle", saisir son rôle dans l'exercice de la mobilité, appréhender les rapports entre les mobiles et les immobiles et "décloisonner » les catégories autour de la mobilité pour révéler des pratiques non pas opposées mais bien complémentaires : comment cette mobilité (et donc les catégories) a-t-elle orienté mes réflexions? Je restituerai la manière dont l'immobilité est apparue initialement dans mes travaux et comment je l'ai appréhendée par la suite. Mais aussi, avec quelles difficultés j'ai pu contourner cette assise de la mobilité. Cet article interroge donc le rôle de l'immobilité dans les déplacements mais aussi dans les trajectoires résidentielles, au regard de deux catégories d'habitat considérées comme instables et éphémères. 


\section{Habitat mobile et immobilité ?}

L'habitat mobile fait partie de la catégorie plus générale des HNO (Habitat Non-Ordinaire) qui se différencie de «l'habitat ordinaire» représentant « le fait d'une part de résider dans le cadre du droit commun, soit en tant que propriétaire, soit en tant que locataire, et d'autre part d'y être chez soi » (Lévy-Vroelant, 2000: 14). L'habitat mobile est alors considéré comme faisant partie des "logements de passage» souvent «confondus dans une même catégorie fonctionnelle, du fait qu'ils sont voués à une fonction d'accueil et de transit » (Lévy-Vroelant, 2004 : 157). Cela sous-tend par conséquent l'idée de mouvement, de départ et d'arrivée. Cet aspect transitoire de la présence sur un territoire questionne particulièrement l'intensité et la fréquence des déplacements qui sont élevés en caractéristique principale au détriment d'autres logiques qui sont minorées mais qui participent à la reproduction de la mobilité, et dont les périodes d'immobilité font partie. Elles sont cependant comme « dénaturées de leurs qualités» et l'immobilité comme ressource n'est que difficilement considérée. La visibilité première étant le type d'habitat (ici mobile) et la mobilité qui y est, de fait, associée. Pourtant, l'immobilité, dans mes recherches et questionnements, s'est révélée être une approche très satisfaisante dans la compréhension de la mobilité.

\section{L’immobilité comme ressource}

La question de la prévalence de la mobilité sur l'immobilité (du point de vue de la recherche) est apparue pendant mon travail de thèse qui portait sur la circulation des Gens du voyage en France. L'objectif était de comprendre au mieux les logiques qui sous-tendaient cette mobilité, de voir l'organisation du parcours et de déceler les motifs de la circulation. Cette focalisation sur la mobilité est très vite apparue comme un écueil puisque elle mettait l'accent sur les déplacements, leurs motifs, mais très peu sur les potentialités des lieux du parcours alors qu'ils accueillaient l'immobilité et étaient utilisés comme des ressources (économiques notamment) pour mieux repartir. Ici, l'immobilité rend possible la mobilité.

\section{L'immobilité comme facilitatrice/initiatrice de la mobilité}

Une des approches de mon travail est de considérer l'immobilité et surtout les immobiles comme des acteurs de la circulation de ceux qui se déplacent. À l'image des analyses qui ont été réalisées sur la place des non-migrants dans les parcours migratoires de ceux qui partent en migration, mes travaux ont également pointé ce phénomène concernant les circulations des Gens du voyage. Par exemple, les lieux de résidence des familles de Gens du voyage « sédentaires» sont investis par celles qui sont encore en circulation. Pour les populations «nomades», la circulation repose, entre autres, sur deux caractéristiques majeures : la dispersion des membres du groupe et la recomposition familiale. Cette dernière présente des périodes d'immobilité où les solidarités et les forces familiales se partagent en vue d'une meilleure emprise sur l'espace pratiqué par les familles.

Il se dessine véritablement un phénomène de pluri-résidence qui s'appuie sur un système résidentiel assez complexe où différents lieux de séjour et d'installation se succèdent. Ce système résidentiel étant animé par des réseaux familiaux importants et fédérateurs de la circulation. Ici, l'immobile est acteur de la circulation.

Ces constats (rôle des immobiles et des périodes d'immobilité sur la circulation) conduisent à remettre en cause deux catégories :

- celle liée au type d'habitat et qui est conditionnée par des pré-supposés liés à la mobilité : un individu qui habite en habitat mobile ne sera pas forcément mobile ; 
- celle liée aux pratiques des individus : périodes d'immobilité et de mobilité peuvent alterner, se compléter ou encore se superposer.

Ces confusions contribuent, de fait, à la création de catégories administratives qui ne considèrent pas la complémentarité de l'immobilité et de la mobilité et qui « fixent» des pratiques (comme c'est le cas pour les catégories administratives «itinérants", «semi-sédentaires» et «sédentaires» qui sont employées pour identifier les Gens du voyage en France). Mes travaux ont révélé que les situations de mobilité et d'immobilité ne sont pas acquises, définitives, elles ne sont pas cloisonnées, et toute tentative de catégorisation appauvrit les analyses que l'on peut faire des pratiques.

La notion de système résidentiel, appliquée à la circulation des Gens du voyage, m'a encouragée à questionner les échelles d'analyse, notamment quand le rôle de la famille est prépondérant. Ainsi, comme le résume Michel Agier (1999: 25), «le rapport de parenté structure la pratique résidentielle ». Selon les individus sur lesquels les analyses sont réalisées, certaines catégories sont plus adéquates. Le système résidentiel est ici entendu comme «un ensemble articulé de lieux de résidence (unités d'habitation) des membres d'une famille étendue ou élargie » (Le Bris et al., 1985: 25). Dans le cas de mes travaux portant sur les Gens du voyage, la cellule familiale élargie est apparue révélatrice des liens existants entre logiques résidentielles et logiques de mobilité. Cette approche m'a ainsi permis de différencier les périodes d'immobilité et de mobilité, et de montrer l'importance des acteurs immobiles dans la reproduction de la circulation puisqu'ils apparaissaient comme faisant partie du système résidentiel bien qu'ils ne circulent pas.

Enfin une dernière dimension mérite une attention particulière puisqu'elle met en évidence une certaine "stabilité résidentielle » au sein même de la mobilité. La régularité des parcours, l'investissement régulier des mêmes lieux et ce d'une année sur l'autre, confèrent une permanence dans la mobilité. Il ne s'agit pas ici d'une immobilité au sens premier du terme; mais l'étude des trajectoires résidentielles, recueillies à l'aide d'entretiens semi-directifs et de matériaux spécifiques concernant les lieux investis par les familles, permet de révéler le caractère répétitif de la circulation. Cela témoigne notamment de l'ancrage des familles dans leur espace de circulation. La mobilité n'empêche donc pas l'ancrage puisque les périodes d'immobilité et la stabilité résidentielle du parcours permettent aux individus de s'inscrire historiquement, socialement, etc., sur un territoire.

\section{Habitat précaire et immobilité}

Le deuxième point de cet article tente d'opérer le croisement entre habitat précaire et pratiques de mobilité/immobilité. Il s'agit d'exposer des perspectives de recherche qui ont été tracées à partir mes travaux antérieurs dans lesquels j’ai focalisé mes analyses sur des «situations résidentielles spécifiques » et discuté cette acception de la spécificité. Une analyse des " comportements résidentiels » a émergé au regard de la mobilité et de l'immobilité des personnes qui ont fait l'objet de ma recherche. Ces observations méritent d'être mises en perspective avec d'autres catégories de la population qui se positionnent aussi dans un environnement résidentiel qui n'entre pas dans les conceptions communes de l'habitat et du logement, et conduisent à travailler sur des trajectoires résidentielles distinctives.

\section{L'habitat précaire : cadrage théorique}

La précarité résidentielle est ici entendue selon quatre critères principaux :

- L'instabilité de la présence : la nondurabilité du logement instaure une mobilité résidentielle ou, tout du moins, un caractère fragile et vulnérable de la présence des 
personnes et du logement. Des trajectoires résidentielles "contraintes» peuvent apparaitre sous l'effet de cette non-durabilité mais aussi sous l'effet d'absence de choix résidentiel, entendu ici comme «un acte important et difficile », qui n'est pas «le fruit du hasard» et qui est dépendant de différents arbitrages opérés par les individus en fonction de leur vie sociale. (Authier, Bonvalet, Lévy, 2010).

- Les matériaux constitutifs des murs, $\mathrm{du}$ toit et $\mathrm{du}$ sol, dont la principale caractéristique est leur fragilité. À ceci s'ajoute les équipements élémentaires tels que l'accès à l'eau et à l'électricité, qui sont souvent absents ou réalisés de façon « artisanale ».

- Le statut des personnes qui habitent ces logements précaires en fonction, en premier lieu, de la classe sociale d'appartenance: je fais l'hypothèse qu'il existe un lien entre précarité résidentielle et précarité économique dans la mesure où «la question des stratégies, résidentielles et autres, est directement liée à celle des ressources [économiques entre autres]. Sans ressources, pas de stratégies » (Dureau, et al., 2000 : 30). Néanmoins, d'autres personnes peuvent se retrouver de façon transitoire dans la "séquence précaire » pour accéder à un logement qui corresponde mieux à leur choix. Donc le lien entre précarité résidentielle et précarité économique n'irait pas de soi, surtout dans les formes contemporaines de la précarité résidentielle. Et enfin, celle de l'illégalité de la présence sur le territoire national (certaines de ces personnes peuvent être en situation irrégulière au regard des lois du pays d'accueil).

- Le statut d'occupation : le logement ou le terrain peut être investi de manière illégale et ces personnes occupent un lieu qui ne leur appartient pas. À l'opposé, les habitants de ces lieux peuvent être locataires ou encore propriétaires.

La précarité résidentielle apparait ces dernières années sur la scène politique et associative sous l'expression du «mal- logement » caractérisé par quatre indicateurs principaux qui peuvent être dissociés les uns des autres et qui recoupent les qualités mentionnées ci-dessus : absence du logement, difficultés d'accès, manque de confort et insalubrité, difficultés de maintien, mobilité difficile, et assignation à résidence (Dietrich, 2007). Toutes ces modalités peuvent se regrouper sous la notion d'« exclusion du logement » qui croise « type d'habitat, statut juridique d'occupation, degré de stabilité ou de précarité d'occupation et qualité du logement» (CNIS, 1996).

\section{La précarité résidentielle au regard de la mobilité et de l'immobilité}

Comment considérer l'immobilité dans le contexte de la précarité résidentielle ? Deux approches peuvent être envisagées :

La première concerne le rôle de la précarité sur les trajectoires résidentielles: cette position résidentielle (qualité du logement, localisation, mode et statut d'occupation), bien qu'instable, engendre-telle inévitablement de l'immobilité résidentielle? Ici se pose avec acuité la notion de choix. Les habitants de ces lieux d'habitat précaire interrogent cette notion qui conditionne en grande partie l'immobilité et la mobilité résidentielle.

La précarité est-elle une contrainte du point de vue des stratégies résidentielles? Engendre-t-elle des pratiques résidentielles spécifiques, moins «dynamiques » que celles d'autres catégories de la population qui se situent dans le cadre légal du logement?

De nombreux travaux, initiés dès la fin des années 1980, ont montré l'existence de stratégies et de choix résidentiels, même pour les catégories de population les plus défavorisées. Pourtant, la question des ressources - notamment économiques-dans la mobilité résidentielle est essentielle. Bien que ces choix résidentiels existent, ils sont réduits et influencent de fait les pratiques 
résidentielles : «Les inégalités s'accentuent à l'échelle métropolitaine et les choix résidentiels semblent très réduits pour les plus modestes : c'est le plus souvent dans la localisation au sein des espaces qui leur sont accessibles que s'expriment leur choix » (Dureau, Bonvalet, 2000 : 153). Dans le cadre de l'habitat précaire, la mobilité résidentielle laisserait donc sa place à une «stabilité résidentielle » dans la mesure où le choix des possibles est réduit pour les individus et les familles qui souhaitent effectuer une mobilité résidentielle. La question du choix est alors «l'entre-deux » entre mobilité et immobilité résidentielle.

Par ailleurs, la question de l'immobilité liée au contexte de l'habitat se pose également dans le cadre d'une mobilité résidentielle subie. Qu'en est-il des familles qui n'ont pas d'autres solutions (choix ?) que de se diriger vers le précaire (souvent illégal) et qui subissent des expulsions? On peut supposer que, à l'instar des difficultés rencontrées par les individus et les familles qui s'inscrivent dans le cadre formel du logement, il existe une tension liée au logement et à l'habitat; l'immobilité résidentielle devient alors pour ces familles le gage de la «possession » d'un toit, bien que celui-ci soit illégal et de ce fait «instable». Ici, l'immobilité est considérée comme une « sécurité» et la mobilité comme une contrainte.

La seconde approche interroge le rôle de la précarité résidentielle sur l'insertion, l'accès aux ressources de la ville, et les pratiques habitantes : la position résidentielle a-t-elle des conséquences sur la mobilité spatiale des individus et les manières de s'établir dans la ville ? Le deuxième lien entre immobilité/mobilité et habitat précaire que je souhaite interroger peut être appréhendé par le biais des pratiques de mobilité (et d'immobilité) dans l'espace urbain qui accueille le lieu de résidence. Il s'agit de voir comment le statut d'occupation (ici précaire et parfois illégal), la localisation et le caractère «instable» de la résidence, influencent d'une part l'usage des services de la ville et, d'autre part, les déplacements à l'intérieur de la ville.

Les recherches que j’ai menées dans le cadre de ma thèse m'ont amenée à m'interroger sur la place du lieu de résidence et/ou de stationnement, qu'il soit éphémère ou pérenne, illégal ou non, dans la ville et ce en contexte de mobilité résidentielle. Une attention spécifique a été portée aux liens entre les habitants de ces espaces, leur voisinage et l'espace urbain. Mes observations ont révélé une certaine dynamique de quartier dans le cadre de l'étude des Gens du voyage ; ce qui n'est pas non plus sans remettre en cause la conception d'espaces résidentiels perçus également comme des espaces enclavés et isolés au sein de l'espace urbain.

Concernant ces lieux d'habitat
précaire, qui m'intéressent plus particulièrement, il s'agit d'examiner et de caractériser les relations entre les positions résidentielles (qualité du logement, localisation, mode et statut d'occupation) et les pratiques habitantes et de voir comment elles interagissent. Il s'agit de se demander en quoi ce qui est considéré comme « marge » fait système ou corps avec la ville. L'observation des pratiques habitantes appréhendées par le biais des pratiques de mobilités/immobilités et de l'accès aux ressources de la ville vont ainsi permettre de révéler si ces lieux a priori enclavés sont connectés ou déconnectés de la ville. 


\section{Regard sur la méthodologie et questionnements transversaux}

\section{La précarité résidentielle et \\ le mal-logement dans les enquêtes liées au logement et dans le recensement de la population}

Le mal-logement et la précarité résidentielle ne sont pas absents des grandes enquêtes statistiques nationales. Dès les années 1990, le Centre National de l'Information Statistique (CNIS) réalise un rapport intitulé «Pour une meilleure connaissance des sans-abri et de l'exclusion du logement» suite aux travaux du groupe sur les sans-abri. Il pointe, entre autres, les difficultés rencontrées dans la production de statistiques concernant des contextes de vie spécifiques et difficiles. En 2001, en collaboration avec l'Ined, l'Insee poursuit les travaux sur cette catégorie de population. La thématique s'ouvre dans les années 2000 au moment où la Commission «Démographie et questions sociales » du CNIS, accorde une importance spécifique au mal-logement dans son acception large. Plusieurs bilans ont été produits, notamment par l'Insee, entre autres celui sur «Les situations marginales par rapport au logement. Méthodes et sources statistiques publiques » en 2009, ou encore celui sur «Les dispositifs statistiques de l'Insee dans le domaine du logement. État des lieux et évaluation comparée des sources » en 2010. Concernant la problématique du mal-logement et de la précarité résidentielle, celle-ci est présente de façon succincte dans l'information méthodologique relative aux Recensements Généraux de la Population de 1990 et 1999. En effet, plusieurs catégories de logements et de lieux peuvent concerner cette problématique, à savoir les catégories "caravanes et autres habitations mobiles", " constructions provisoires et habitations de fortune », "squats », et "hôtels ». Dans la version rénovée du recensement de 2004, une des priorités est donnée à l'identification des situations de mal-logement et de précarité résidentielle. Ce souci de clarification répond, entre autres, à des lacunes du recensement concernant ce type particulier du parc du logement: «les situations marginales de logement constituent une des plus grandes difficultés et sources d'erreurs du recensement» (Coeffic, 1993 : 1666). Un des objectifs du recensement rénové est donc de mieux prendre en compte ces situations résidentielles spécifiques tout en continuant des partenariats avec d'autres organismes dont l'Ined - sur cette thématique; le rapport d'Alexandre Djirikian, Valérie Laflamme et Maryse Marpsat réalisé en 2006 sur « les situations marginales de logement» et plus précisément sur la nécessité de mieux cadrer et définir ces situations, va en ce sens. Enfin, les enquêtes logement - qui constituent la source statistique principale concernant le parc du logement en France et les conditions d'occupation des résidences principales - ne prennent en compte que très partiellement ces situations résidentielles spécifiques dans la mesure où des catégories concernées par le mal-logement et la précarité résidentielle ne sont pas retenues, comme celles par exemple des «caravanes et habitations mobiles » (considérées comme des $\mathrm{HNO}{ }^{1}$, ou encore les «habitations de fortune et les constructions provisoires ». En effet, la base SITADEL (Système d'Information et de Traitement Automatisé des Données Élémentaires sur les Logements et les locaux) utilisée pour identifier les lieux d'enquête, ne se concentre que sur les habitations ayant obtenu un permis de construire. En ce sens, les constructions de fortune comme les cabanes, etc., ne sont pas identifiées comme un lieu d'enquête.

Pour toutes ces raisons, la nécessité de travailler plus précisément sur le mallogement, la précarité résidentielle et les situations de vie qui en découlent est intéressante. Les séquences précaires, dans l'ensemble de la carrière résidentielle, sont difficilement saisissables. Les rapports

\footnotetext{
${ }^{1}$ Seules les caravanes immobilisées (c'est-à-dire dont les roues ont été enlevées) sont prises en compte dans l'enquête logement.
} 
effectués sur la place de cette problématique, que cela soit dans les grandes enquêtes nationales ou dans le recensement, montrent plusieurs lacunes, notamment un problème d'homogénéisation du vocabulaire qui entraîne des difficultés de caractérisation des situations de mal-logement ainsi qu'une absence ou une "mauvaise » production de la statistique liée à ces contextes résidentiels.

\section{Situations résidentielles spécifiques et collecte des données}

Dans mes travaux de thèse, la question de la méthodologie autour des pratiques de mobilité et de l'immobilité s'est évidemment posée avec acuité. Il est très vite apparu que réaliser des enquêtes biographiques était très difficile, notamment du fait de la fréquence des déplacements, et ce sur un temps relativement court. Les lieux de vie au cours de l'année étaient multiples et réinvestis parfois plusieurs fois sur ce même pas de temps. Une difficulté supplémentaire a aussi rapidement suggéré de réorienter l'approche méthodologique : les Voyageurs activaient le souvenir par l'évènement et non par la date, ce qui rendait véritablement compliquée l'utilisation de ce type de support. Par ailleurs, une difficulté supplémentaire est apparue lorsqu'il a été question de cartographier les itinéraires des Gens du voyage rencontrés. Si le parcours était facilement représentable, les périodes d'immobilité, et surtout celles qui supposaient une discontinuité, une rupture dans le parcours, posaient problème.

Dans le cadre de mes travaux actuels sur ces lieux d'habitat précaire, le lien entre immobilité et mobilité, tant du point de vue des trajectoires résidentielles que des mobilités quotidiennes, est abordé à plusieurs échelles. À l'échelle internationale, aucun parti pris n'est fait à propos des catégories sociales des personnes qui habitent ces espaces mais la présence de populations migrantes pourra introduire cette échelle d'analyse ; puis à l'échelle interurbaine, une des hypothèses est que si la mobilité résidentielle existe, elle peut se faire à l'échelle de plusieurs villes; et enfin à l'échelle infra-urbaine, les trajectoires résidentielles ainsi que les pratiques de l'espace urbain pourront être appréhendées spécifiquement dans le cadre d'une analyse à l'échelle de la ville qui accueille le lieu de résidence au moment de l'étude.

Enfin, les analyses longitudinales sont un moyen de saisir les immobilités résidentielles dans la mesure où elles permettent d'avoir une observation des mobilités sur un temps long.

\section{Conclusion}

Les questionnements soulevés dans cette introduction sont transversaux et font écho à l'ensemble des contributions de ces deux journées. Quatre d'entre eux méritent particulièrement d'être mentionnés, dans un premier temps celui concernant la méthodologie: des réflexions autour de la collecte, l'analyse et la retranscription de l'immobilité paraissent fondamentales. Dans un second temps, il apparait nécessaire d'interroger - voire de remettre en cause et de faire évoluer - les catégories qui peuvent avoir une influence sur les matériaux d'enquête mais aussi à une autre échelle, sur la gestion territorialisée des pratiques et des populations). Un autre questionnement nous mènera à discuter la traditionnelle dichotomie mobilité/immobilité (en quoi les pratiques forment-elle un tout, sont interdépendantes, et participent l'une et l'autre à la reproduction des pratiques de mobilité et d'immobilité). L'axe MIT (Mobilité, Identité et Territoire) du CIST insiste sur les identités territorialisées en situation de mobilité : qu'en est-il du lien immobilité, identité et territoire?

Céline Bergeon ATER Université de Limoges CNRS - UMR 7301 Migrinter bergeonceline@yahoo.fr 


\section{Bibliographie}

Agier, Michel. (1999) L'invention de la ville: banlieues, townships, invasions et favelas, Paris, Éd. des archives contemporaines, 176 p.

Authier, Jean-Yves; Bonvalet, Catherine; Lévy, Jean-Pierre (2010) Elire domicile: la construction sociale des choix résidentiels, Lyon, Presse Universitaire de Lyon, 434 p.

Coeffic, Nicole (1993) L'enquête postcensitaire de 1990: une mesure de l'exhaustivité du recensement, Population, n6, pp. 1655-1682.

Dietrich-Ragon, Pascale (2007) Tensions autour des procédures liées à l'insalubrité. L'exemple du saturnisme infantile, in Laflamme, V. (dir) Le logement précaire en Europe, Aux marges du palais, Paris, L'Harmattan, pp. 347-360 (Habitat et sociétés).

Dureau, Françoise; Dupont, Véronique ; Lelièvre, Eva; Lévy, Jean-Pierre ; Lulle, Thierry (dir.) (2000) Métropoles en mowvement, une comparaison internationale, Paris, Anthropos, 656 p.

Le Bris, Emile; Marie, Alain ; Osmont, Annik; Sinou, Alain (1987) Famille et résidence dans les villes africaines. Dakar, Bamako, SaintLouis, Lomé, Paris, L'Harmattan, 268 p. (Villes et entreprises).

Lévy-Vroelant, Claire (2000) Logements de passage, normes, formes, expériences, Paris, l'Harmattan, 297 p.

Lévy-Vroelant, Claire (2004) Le logement des migrants en France du XIXe siècle à nos jours, Historiens et Géographes, n³85, pp.147165. 